\title{
New syntaxa of tall-forb vegetation in the Pamir-Alai and western Tian Shan Mts. (Tajikistan and Kyrgyzstan, Middle Asia) - an addendum to Nowak et al. 2020
}

\author{
Arkadiusz Nowak ${ }^{1,2}$, Sebastian Świerszcz ${ }^{1,3}$, Sylwia Nowak ${ }^{2}$, Marcin Nobis ${ }^{4,5}$ \\ 1 Botanical Garden, Center for Biological Diversity Conservation, Polish Academy of Sciences, Warszawa, Poland \\ 2 Institute of Biology, University of Opole, Opole, Poland \\ 3 The Franciszek Górski Institute of Plant Physiology, Polish Academy of Sciences, Kraków, Poland \\ 4 Department of Taxonomy, Phytogeography and Palaeobotany, Institute of Botany, Jagiellonian University, Kraków, Poland \\ 5 Research laboratory 'Herbarium', National Research Tomsk State University, Tomsk, Russia
}

Corresponding author: Sebastian Świerszcz (s.swierszcz@obpan.pl)

Academic editor: Wolfgang Willner • Received 26 August 2021 • Accepted 9 September 2021 • Published 25 October 2021

\begin{abstract}
We validate eleven syntaxa (eight associations and three alliances) of tall-forb vegetation that were published earlier as nomina provisoria according to the International Code of Phytosociological Nomenclature. The validation concerns syntaxa of tall-forb vegetation of the class Prangetea ulopterae Klein 1987 reported from Pamir-Alai and western Tian Shan Mountains (Tajikistan and Kyrgyzstan).
\end{abstract}

Taxonomic reference: Cherepanov (1995).

Abbreviations: ICPN = International Code of Phytosociological Nomenclature.

\section{Keywords}

International Code of Phytosociological Nomenclature (ICPN), Middle Asia, phytosociological nomenclature, Prangetea ulopterae, syntaxonomy, tall forb, validation

\section{Introduction}

According to the older version of the International Code of Phytosociological Nomenclature (ICPN, Weber et al. 2000), an effectively published syntaxon should be distributed as a printed matter to the general public or at least to libraries accessible to botanists (Article 1 ICPN). This is related also to the date of the valid publication and to the rule of priority (Articles 2, 6, 23 ICPN). In the last few years, many publishers decided to broadcast parts of papers (e.g. tables, large data sets, pictures, figures) or whole journals online only, without printed versions. This causes some difficulties, particularly with effective publication of names of newly established syntaxa that had to be regarded as nomina inedita if the paper was entirely published on-line, or as nomen nudum, if the typus relevés were distributed only as online appendix (nevertheless it has a digital identifier). This is why the latest version of the code already provides the possibility of valid publishing of the syntaxon name in an electronic version of the paper, which does not appear as a printed copy. An electronic publication is accepted as effective on or after 1 January 2021 only in the form of Portable Document Format (PDF) that 
would bear either an International Standard Book Number (ISBN) or an International Standard Serial Number (ISSN) or a Digital Object Identifier (DOI) (Theurillat et al. 2021).

As a result of the phytosociological survey in alpine and subalpine belts of Pamir Alai and Tian Shan Mts. in Middle Asia, eleven new syntaxa of tall-forb vegetation were described with the provisional names (Nowak et al. 2020). We originally assumed that the paper will be published in 2021 . However, due to the fast reviewing and editing process, the article was already published in 2020. Therefore, in agreement with the Editors of VCS, we decided that we will publish the new syntaxa as nomina provisoria and validate them in 2021 in accordance with the regulations of the ICPN.

The aim of this paper is to validate the provisional names of tall-forb syntaxa according to the current ICPN and to present the overview of the vegetation types that were described in the first paper devoted to tall-forbs of Middle Asia (Nowak et al. 2020).

\section{Validation of syntaxa}

The numbers of syntaxa refer to the synopsis at the end of the paper. Diagnostic taxa are provided in Nowak et al. (2020).

\section{Forb rich mesophilous tall-forbs of the western Pamir-Alai Mts.}

\subsection{Alliance: Ligulario thomsonii-Geranion regelii Nowak et al. all. nov. hoc loco}

Holotypus: Eremuretum kaufmannii Nowak et al. ass. nov. hoc loco (see below)

\subsubsection{Heracleetum lehmannianii Nowak et al. ass. nov.} hoc loco

Holotypus: 02 July $2017 ; 40.24556^{\circ} \mathrm{N}, 73.34028^{\circ} \mathrm{E} ; 2157 \mathrm{~m}$ a.s.l.; aspect $\mathrm{N}$; slope $3^{\circ}$; plot area $10 \mathrm{~m}^{2}$; cover herb layer 90\%; cover moss layer 3\%; species richness: 28 .

Herb layer: Geranium regelii 3, Euphorbia lamprocarpa 3, Achillea millefolium 2, Carum carvi 2, Dactylis glomerata 2, Poa pratensis 2, Ranunculus brevirostris 2, Heracleum lehmannianum 2, Amoria repens 1, Convolvulus arvensis 1, Cousinia pseudarctium 1, Festuca rubra 1, Galium turkestanicum 1, Glycyrrhiza glabra 1, Hordeum turkestanicum 1, Ligularia thomsonii 1, Mentha asiatica 1, Nepeta cataria 1, Plantago lanceolata 1, Codonopsis clematidea + , Cynoglossum viridiflorum + , Erigeron allochrous + , Lithospermum officinale + , Polygonum coriarium + , Potentilla pedata + , Trifolium pratense +, Vicia tenuifolia +. Moss layer: Ceratodon purpureus 1. [relevé number in Nowak et al. (2020): supplementary material 1: 40]
2.1.4. Eremuretum kaufmannii Nowak et al. ass. nov. hoc loco

Holotypus: 23 June 2014; $39.25722^{\circ} \mathrm{N}, 68.17833^{\circ} \mathrm{E}$; $2836 \mathrm{~m}$ a.s.l.; aspect $\mathrm{N}$; slope $25^{\circ}$; plot area $10 \mathrm{~m}^{2}$; cover herb layer 95\%; species richness: 17; species composition:

Herb layer: Geranium regelii 3, Myosotis alpestris 3, Ligularia thomsonii 2, Poa urssulensis 2, Artemisia dracunculus 1, Astragalus saratagius 1, Carex turkestanica 1, Eremurus kaufmannii 1, Pedicularis grigorjevii 1, Tulipa ingens 1, Veronica rubrifolia 1, Alopecurus pratensis +, Astragalus nuciferus + , Myosotis micrantha + , Nepeta podostachys +, Oxytropis capusii +, Ziziphora pamiroalaica +. [relevé number in Nowak et al. (2020): supplementary material 1: 127]

2.1.5. Anthriscidetum glacialis Nowak et al. ass. nov. hoc loco

Holotypus: 16 June $2019 ; 38.65522^{\circ} \mathrm{N}, 70.73306^{\circ} \mathrm{E} ; 2762 \mathrm{~m}$ a.s.l.; aspect W; slope $10^{\circ}$; plot area $10 \mathrm{~m}^{2}$; cover herb layer $100 \%$; species richness: 33 .

Herb layer: Anthriscus glacialis 3, Geranium regelii 2, Ligularia thomsonii 2, Paeonia intermedia 2, Polygonum hissaricum 2, Astragalus aksuensis 1, Carex dimorphotheca 1, Crepis darvazica 1, Dactylis glomerata 1, Euphorbia glomerulans 1, Fritillaria regelii 1, Leonurus turkestanicus 1, Myosotis alpestris 1, Phleum pratense 1, Poa pratensis 1, Polygonum coriarium 1, Amoria repens +, Astragalus adpressipilosus +, Astragalus tecti-mundi + , Calamagrostis pseudophragmites + , Crocus korolkowii +, Euphorbia sarawschanica +, Galium aparine +, Ligularia alpigena + , Lolium cuneatum + , Medicago romanica + , Oberna wallichiana + , Pedicularis olgae + , Picris nuristanica + , Potentilla sericea + , Rumex nepalensis + , Scilla puschkinioides + , Taraxacum sp. + . [relevé number in Nowak et al. (2020): supplementary material 1:75]

\section{Scree-like tall-forb communities of the eastern Irano-Turanian region}

\subsection{Alliance: Rheion maximoviczii Nowak et al. all nov. hoc loco}

Holotypus: Eremuretum stenophyllido-comosi Nowak et al. ass. nov. hoc loco (see below)

\subsubsection{Phlomoidetum kaufmannianae Nowak et al. ass.} nov. hoc loco

Holotypus: 29 May $2015 ; 39.20556^{\circ} \mathrm{N}, 67.81694^{\circ} \mathrm{E} ; 1715 \mathrm{~m}$ a.s.l.; aspect W; slope $15^{\circ}$; plot area $10 \mathrm{~m}^{2}$; cover herb layer 80\%; cover moss layer 10\%; species richness: 25 .

Herb layer: Phlomoides kaufmanniana 3, Papaver pavoninum 2, Anisantha tectorum 1, Artemisia rutifolia 1, Crambe kotschyana 1, Crepis pulchra 1, Ferula kokanica 1, 
Ferula ovina 1, Piptatherum kokanicum 1, Scandix stellata 1, Taeniatherum crinitum 1, Alcea nudiflora +, Alyssum calycinum + , Arabis recta + , Arenaria serpyllifolia + , Cerastium inflatum +, Erodium cicutarium +, Galium aparine +, Galium spurium +, Geranium divaricatum +, Lappula consanguinea + , Perovskia virgata + . Moss layer: Bryum caespiticium 1, Pohlia nutans 1, Encalypta vulgaris +. [relevé number in Nowak et al. (2020): supplementary material 1: 136]

2.2.5. Eremuretum stenophyllido-comosi Nowak et al. ass. nov. hoc loco

Holotypus: 22 May 2019; $38.70359^{\circ} \mathrm{N}, 70.46913^{\circ} \mathrm{E} ; 1667 \mathrm{~m}$ a.s.l.; aspect SW; slope $40^{\circ}$; plot area $10 \mathrm{~m} 2$; cover shrub layer 15\%; cover herb layer 70\%; species richness 40 .

Shrub layer: Cerasus verrucosa 2, Rosa popovii 2. Herb layer: Eremurus comosus 2, Ferula kuhistanica 2, Prangos pabularia 2, Phlomoides lehmanniana 1, Vinca erecta + , Achillea biebersteinii r, Alcea nudiflora r, Anisantha sterilis r, Antonina debilis r, Asparagus neglectus r, Asperula setosa $\mathrm{r}$, Astragalus macronyx $\mathrm{r}$, Bromus lanceolatus $\mathrm{r}$, Bunium persicum r, Callipeltis cucullaris $\mathrm{r}$, Centaurea squarrosa $\mathrm{r}$, Convolvulus arvensis $\mathrm{r}$, Dianthus darvazicus r, Diarthron vesiculosum $\mathrm{r}$, Dichasianthus subtilissimus r, Eremurus stenophyllus r, Euphorbia franchetii r, Euphorbia sarawschanica r, Galium spurium r, Hordeum bulbosum r, Lactuca tatarica $\mathrm{r}$, Lallemantia royleana $\mathrm{r}$ Lappula consanguinea $\mathrm{r}$, Papaver litwinowii r, Poterium polygamum r, Rheum maximowiczii r, Rhinopetalum bucharicum r, Salvia sclarea r, Serratula chartacea r, Strigosella trichocarpa r, Tanacetum pseudachillea r, Taraxacum nuratavicum r, Veronica rubrifolia r. [relevé number in Nowak et al. (2020): supplementary material 1: 186]

Dry tall-forb communities of the subhumid zone of the eastern Irano-Turanian region

\subsection{Alliance: Scabioso songaricae-Phlomoidion leh- mannianae Nowak et al. all. nov. hoc loco}

Holotypus: Phlomoido lehmannianae-Onobrychidetum grandis Nowak et al. ass. nov. hoc loco (see below)

2.3.3. Lathyretum mulkaki Nowak et al. ass. nov. hoc loco

Holotypus: 17 June 2019; $38.58247^{\circ} \mathrm{N}, 70.76009^{\circ} \mathrm{E}$; $2640 \mathrm{~m}$ a.s.l.; aspect SW; slope $25^{\circ}$; plot area $10 \mathrm{~m}^{2}$; cover shrub layer $2 \%$; cover herb layer $75 \%$; species richness: 31 .

Shrub layer: Rosa ovczinnikovii 1. Herb layer: Ferula kokanica 3, Dictamnus angustifolius 2, Lathyrus mulkak 2, Senecio franchetii 2, Bunium persicum 1, Centaurea ruthenica 1, Delphinium batalinii 1, Nepeta ucranica 1, Poa bulbosa 1, Scabiosa songarica 1, Thalictrum kuhistanicum 1, Ziziphora pamiroalaica 1, Allium hissaricum +, Asperula setosa + , Colchicum luteum + , Cousinia pseudarctium +, Dianthus darvazicus +, Galium spurium +, Hypericum scabrum + , Iris darwasica + , Lappula occultata + , Lophanthus elegans + , Nepeta podostachys + , Origanum tyttanthum + , Phlomoides arctifolia + , Poa zaprjagajevii + , Pseudoclausia turkestanica + , Rhinopetalum bucharicum + , Thymus seravschanicus +, Vinca erecta +. [relevé number in Nowak et al. (2020): supplementary material 1: 90]

\subsubsection{Eremuretum robusti Nowak et al. ass. nov. hoc loco}

Holotypus: 05 June $2015 ; 38.82000^{\circ} \mathrm{N}, 70.20778^{\circ} \mathrm{E} ; 1436 \mathrm{~m}$ a.s.l.; plot area $10 \mathrm{~m}^{2}$; cover herb layer $100 \%$; species richness: 23.

Herb layer: Phlomoides arctifolia 3, Vicia tenuifolia 3, Galium pamiroalaicum 2, Phlomoides lehmanniana 2, Eremurus comosus 1, Eremurus robustus 1, Eremurus stenophyllus 1, Hordeum bulbosum 1, Pimpinella peregrina 1, Poa bulbosa 1, Poa pratensis 1, Potentilla transcaspia 1, Poterium lasiocarpum 1, Prangos pabularia 1, Arenaria serpyllifolia +, Arum korolkowii +, Cousinia pseudarctium +, Galium aparine +, Orobanche sulphurea +, Plantago lanceolata + , Salvia sclarea + , Taraxacum sp. + , Torilis arvensis +. [relevé number in Nowak et al. (2020): supplementary material 1: 179]

2.3.8. Phlomoido lehmannianae-Onobrychidetum grandis Nowak et al. ass. nov. hoc loco

Holotypus: 14 June $2019 ; 38.84710^{\circ} \mathrm{N}, 71.30857^{\circ} \mathrm{E} ; 2519 \mathrm{~m}$ a.s.l.; aspect S; slope $30^{\circ}$; plot area $10 \mathrm{~m}^{2}$; cover shrub layer 5\%; cover herb layer 100\%; species richness: 26 .

Shrub layer: Rosa ovczinnikovii 2. Herb layer: Ferula kuhistanica 3, Phlomoides lehmanniana 3, Prangos pabularia 3, Onobrychis grandis 2, Scabiosa songarica 2, Carex turkestanica + , Poa bulbosa + , Alyssum calycinum $\mathrm{r}$, Arenaria serpyllifolia r, Asperula setosa r, Drepanocaryum sewerzowii r, Elaeosticta allioides r, Gentiana olivieri r, Handelia trichophylla r, Hypericum scabrum r, Lappula patula r, Neurotropis kotschyana r, Poa zaprjagajevii r, Polygonum paronychioides r, Polygonum polycnemoides $r$, Rochelia cardiosepala r, Serratula chartacea r, Stellaria alsinoides r, Taraxacum sp. r, Veronica cardiocarpa r. [relevé number in Nowak et al. (2020): supplementary material 1:242]

\section{Synopsis of the tall-forb communities of the Pa- mir-Alai and western Tian Shan Mountains}

This overview follows the classification scheme of Nowak et al. (2020).

\section{Mesic mown and grazed subalpine meadows and pas- tures on fertile soils}

Class: Molinio-Arrhenatheretea Tx. 1937

1. Order: Poo alpinae-Trisetetalia Ellmauer et Mucina 1993

1.1. Alliance: Poion alpinae Gams ex Oberd. 1950

1.1.1. Community of Phlomoides oreophila 
Irano-Turanian thermophilous, mesic tall-forb communities of the western Pamir-Alai and Tian Shan Mountains

Class: Prangetea ulopterae Klein 1987.

Forb rich mesophilious tall-forb communities of the western Pamir-Alai Mountains

2.1. Alliance: Ligulario thomsonii-Geranion regelii Nowak et al. 2021 all. nov.

2.1.1. Community of Allium hymenorhizum

2.1.2. Feruletum sumbuli Nowak et al. 2015

2.1.3. Heracleetum lehmannianii Nowak et al. 2021 ass. nov.

2.1.4. Eremuretum kaufmannii Nowak et al. ass. 2021 ass. nov.

2.1.5. Anthriscidetum glacialis Nowak et al. 2021 ass. nov.

Scree-like tall-forb communities of the eastern Irano-Turanian region

2.2. Alliance: Rheion maximoviczii Nowak et al. all. 2021 all. nov.

2.2.1. Community of Cousinia batalinii and Euphorbia pamirica

2.2.2. Phlomoidetum kaufmannianae Nowak et al. 2021 ass. nov.

2.2.3. Phlomoidetum tadshikistanicae Nowak et al. 2016 nom. corr. (= Eremostachyetum tadschikistanicae Nowak et al. 2016)

2.2.4. Community of Senecio saposhnikovii

\section{References}

Cherepanov SK (1995) Plantae Vasculares URSS [Vascular plants of the Soviet Union]. Nauka, Leningrad, RU, 532 pp. [In Russian]

Nowak A, Nowak S, Nobis M, Nobis A (2016) Vegetation of screes of the montane and colline zones in the Pamir-Alai Mts in Tajikistan (Middle Asia). Tuexenia 36: 223-248.

Nowak A, Świerszcz S, Nowak S, Nobis M (2020) Classification of tallforb vegetation in the Pamir-Alai and western Tian Shan Mountains (Tajikistan and Kyrgyzstan, Middle Asia). Vegetation Classification and Survey 1: 191-217. https://doi.org/10.3897/VCS/2020/60848
2.2.5. Eremuretum stenophyllido-comosi Nowak et al. 2021 ass. nov.

Dry tall-forb communities of the subhumid zone of the eastern Irano-Turanian region

2.3. Alliance: Scabioso songaricae-Phlomoidion lehmannianae Nowak et al. 2021 all. nov.

2.3.1. Community of Inula macrophylla

2.3.2. Stipetum margelanicae Nowak et al. 2016

2.3.3. Lathyretum mulkaki Nowak et al. 2021 ass. nov.

2.3.4. Potentillo orientalis-Eremuretum fusci Świerszcz et al. 2020

2.3.5. Hordeo bulbosi-Astragaletum retamocarpi Świerszcz et al. 2020

2.3.6. Community of Ferula kuhistanica

2.3.7. Eremuretum robusti Nowak et al. 2021 ass. nov.

2.3.8. Phlomoido lehmannianae-Onobrychidetum grandis Nowak et al. 2021 ass. nov.

\section{Author contributions}

A.N. led the writing, while all authors critically revised the final manuscript.

\section{Acknowledgements}

The authors are grateful to Wolfgang Willner for providing valuable comments on the text.

Theurillat J-P, Willner W, Fernández-González F, Bültmann H, Čarni A, Gigante D, Mucina L, Weber H (2021) International Code of Phytosociological Nomenclature. $4^{\text {th }}$ edn. Applied Vegetation Science 24: e12491. https://doi.org/10.1111/avsc.12491

Weber HE, Moravec J, Theurillat J-P (2000) International Code of Phytosociological Nomenclature. $3^{\text {rd }}$ edn. Journal of Vegetation Science 11: 739-768. https://doi.org/10.2307/3236580

\section{E-mail and ORCID}

Arkadiusz Nowak (anowak@uni.opole.pl), ORCID: https://orcid.org/0000-0001-8638-0208

Sebastian Świerszcz (Corresponding author, seb.swierszcz@gmail.com), ORCID: https://orcid.org/0000-0003-2035-0035 Sylwia Nowak (snowak@uni.opole.pl), ORCID: https://orcid.org/0000-0003-2867-9839

Marcin Nobis (m.nobis@uj.edu.pl), ORCID: https://orcid.org/0000-0002-1594-2418 